\title{
When does a granular material behave like a continuum fluid?
}

\author{
John R. de Bruyn $\dagger$ \\ Department of Physics and Astronomy, University of Western Ontario, London, \\ Ontario, N6G 2R1, Canada
}

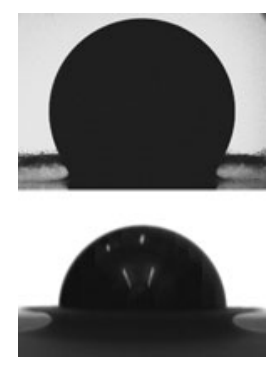

\begin{abstract}
A flowing granular material can behave like a collection of individual interacting grains or like a continuum fluid, depending in large part on the energy imparted to the grains. As yet, however, we have no general understanding of how or under what conditions the fluid limit is reached. Marston, Li \& Thoroddsen (J. Fluid Mech., this issue, vol. 704, 2012, pp. 5-36) use high-speed imaging to investigate the ejection of grains from a granular bed due to the impact of a spherical projectile. Their high temporal resolution allows them to study the very fast processes that take place immediately following the impact. They demonstrate that for very fine grains and high impact energies, the dynamics of the ejecta is both qualitatively and quantitatively similar to what is seen in analogous experiments with fluid targets.
\end{abstract}

Key words: granular media, fluidized beds, particle/fluid flows

\section{Introduction}

Granular materials play a role in almost every aspect of our lives, and are important in agriculture, pharmaceuticals, construction, and many other industries. The properties of a granular material can vary dramatically. Sand on a beach is solid-like, but sand on a hillside can flow - sometimes catastrophically - if the conditions are right. If a container filled with many small particles is shaken, the granular system will behave as a solid, liquid, or gas depending on how hard you shake. To complicate matters, granular flows generally differ from conventional fluid flows because of packing effects and the strong dissipation that results from friction and collisions among the grains. There is as yet no general theory that describes the flow behaviour of granular systems, and the question of when a collection of grains can be adequately described as a continuum fluid is a major open issue in the field. One interesting and complex granular flow results from the impact of a falling projectile into a granular target. Portions of the granular material are fluidized by the impact, with grains initially being forced outwards, then collapsing back inwards as the projectile penetrates below the surface. In addition, some grains are ejected from the target in a process analogous to the familiar formation of a splash in liquids. The end result is an impact crater similar to those seen on the Moon and rocky planets. The flow is transient and evolves very quickly, making it challenging to study.

Early work on granular impacts was motivated by an interest in the formation of planetary craters. Several recent papers have studied the morphology and scaling of granular craters (Uehara et al. 2003; Walsh et al. 2003; de Vet \& de Bruyn 2007)

$†$ Email address for correspondence: debruyn@uwo.ca 


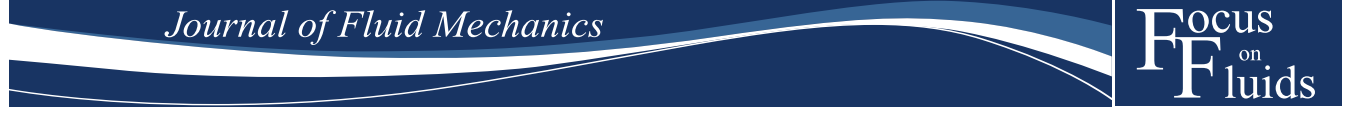

(a)

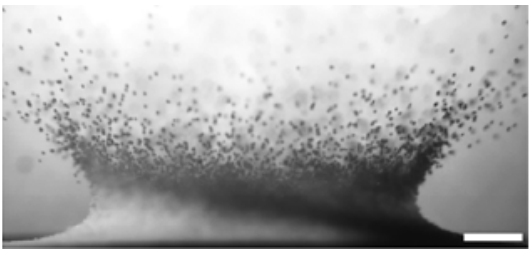

(b)

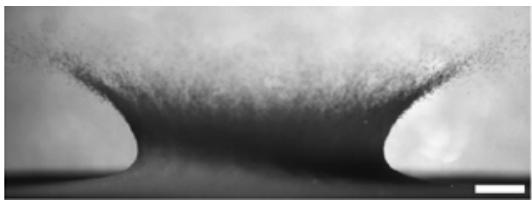

(c)

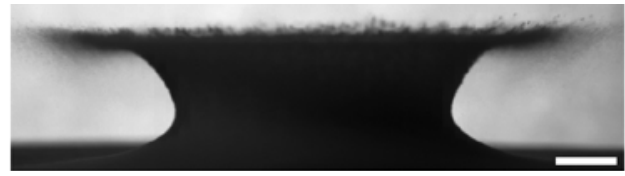

(d)

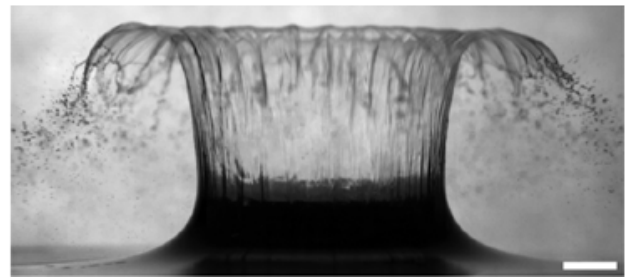

FIGURE 1. Video images showing the ejecta produced by the impact of a solid sphere onto targets of: (a) $520 \mu \mathrm{m}$ glass beads; (b) $178 \mu \mathrm{m}$ glass beads; (c) $31 \mu \mathrm{m}$ glass beads; and $(d)$ water. The size of the sphere and the impact velocity were the same in all cases. While the granular nature of the ejecta is evident in $(a)$, the flow for the finest spheres looks qualitatively very similar to that for water. (From Marston et al. 2012: scale bars are $1 \mathrm{~cm}$.)

as well as the granular flows involved in their formation (de Vet et al. 2010). The dynamics of the impact and the penetration of the projectile into the target has been studied as a probe of the forces exerted by the granular medium on the projectile (de Bruyn \& Walsh 2004; Ambroso, Kamien \& Durian 2005; Katsuragi \& Durian 2007; Goldman \& Umbanhowar 2008). In addition, impact into a target of fine, loosely packed grains can produce impressive granular jets (Thoroddsen \& Shen 2001) that are analogous to the well-known Worthington jets seen in fluid impacts.

A recent paper by Marston, Li \& Thoroddsen (2012, this issue) reports a detailed investigation of the very early stages of the granular impact process, focusing on the ejection of grains from the target. Marston et al. (2012) use high-speed imaging to study the appearance and early evolution of the ejecta with a time resolution of up to $10 \mu \mathrm{s}$, much better than that in previous studies of granular ejecta (Boudet, Amarouchene \& Kellay 2006; Deboeuf, Gondret \& Rabaud 2009). This allows them to view the very rapid events that take place immediately following the impact. In addition to more fully characterizing this complex granular flow, their research helps to address the question of when a granular flow displays truly fluid behaviour.

\section{Overview}

Marston et al. (2012) studied the ejection of material caused by the impact of steel spheres of a range of diameters and impact speeds into targets consisting of small spherical glass beads or sand grains. Images $(a-c)$ in figure 1 , which is taken from their paper, are snapshots of the curtain of granular ejecta produced by impact into targets of different sized glass beads. The last image (figure $1 d$ ) shows the fluid ejecta following an impact into water. An evolution of the granular ejecta towards more fluid-like appearance and behaviour is evident as the grains get smaller. The Froude number $\left(F r=V_{0} / \sqrt{g D_{0}}\right.$, where $D_{0}$ is the sphere diameter, $V_{0}$ its impact speed, and $g$ the acceleration due to gravity) is the same in all four cases illustrated in figure 1, as is the dimensionless time from the sphere's first contact with the target material. The packing fraction of the grains and the ratio of $D_{0}$ to the diameter of the grains 
are different. The shape and size distributions of the grains also play a role. Marston et al. systematically characterize the effects of these parameters on the ejecta.

Experiments with targets made of larger glass beads display an 'early stage' in which individual grains are ejected prior to the development of a coherent ejecta sheet in the 'main stage'. The first ejected grains appear within a few milliseconds of the initial contact of the sphere with the target. Although seemingly fast, this is an order of magnitude longer than the time for the splash to appear when a sphere hits a fluid (Thoroddsen et al. 2004). Marston et al. suggest that this delay is due the compressibility of the granular material, which reduces the propagation speed of the disturbance produced by the sphere. This effect is even larger for sand grains, which are less spherical and have a broader shape distribution. When distances are scaled by the sphere diameter $D_{0}$ and times by the reciprocal of the local shear rate $V_{0} / D_{0}$, both the time and the radial position at which the grains emerge are independent of $F r$, and largely independent of both the size ratio and the packing fraction.

The high-speed imaging technique used by Marston et al. gives them access to the previously unobserved early stage of the ejection process. Streakline images of individual grains ejected before the formation of the main ejecta sheet allow direct determination of the angle and speed of ejection for each grain; over the time range studied air drag is almost negligible and the grains travel in straight lines. The fastest grains can be ejected with a speed five times that of the impacting sphere. In a given experiment, the grains ejected at early times are on average faster and are emitted at lower angles than at later times, although there is a large range of both speed and angle due to collisions among the particles as they make their way out of the bulk material. The evolution of the ejection angle is simply explained by the change in the angle between the surface of the sphere and that of the target as the sphere penetrates more deeply, and the range of angles and speeds narrows with time as the ejected grains converge to a fluid-like sheet.

In impacts into the finest grains, in contrast, the ejecta forms a coherent sheet, similar in appearance to that seen in impacts into water, from the earliest observable times. The velocity of the emerging sheet is proportional to the impact velocity and increases with the sphere diameter, but is independent of the packing fraction. The tip of the ejecta sheet thickens with time, but air resistance causes individual grains to break off from the tip, forming a hazy cloud around the sheet. The dynamics of this sheet can become quite complex due to an interplay among air entrainment, a vortex ring generated inside the ejecta curtain, and the porosity of the sheet itself. While the packing fraction of the granular target has little effect on the early stages of ejecta formation, it does affect the later evolution of the sheet, as higher packing fractions lead to more porous ejecta sheets.

Marston et al. tracked the location of the narrowest point of the coherent ejecta sheet as a measure of its expansion with time. They found a power-law scaling of the neck radius at early times, but with a non-universal exponent. Interestingly, the exponents they found are all much lower than the value of 2 predicted by a model due to Deboeuf et al. (2009). Marston et al. speculate that the early-time behaviour they observe is quite different from the later-time dynamics treated previously, and point to indications that their exponent may in fact be approaching 2 at later times. For the lowest packing fractions, finest grains, and largest impact energies, the exponent measured by Marston et al. is close to the value of 0.5 seen in the fluid ejecta sheets produced by impacts into liquid films. This scaling, along with the fluid-like appearance of the sheet seen with the finest grains, suggest that the granular ejecta sheet approaches true fluid-like behaviour in the fine-particle limit. 
There are some differences between these granular ejecta sheets and those sheets seen in fluid impact experiments. For example, for similar values of the effective Reynolds number, the velocity of the ejecta sheet is roughly a factor of 2 smaller for granular impacts than in the fluid case. Marston et al. (2012) suggest that such differences may be resolved by a better understanding of the effective viscosity of the granular bed, and the changes in packing fraction that occur in response to the impact.

\section{Future}

The work of Marston et al. (2012) gives us a much more complete picture of the ejection of grains in a granular impact and demonstrates the approach of this particular granular flow to fluid-like behaviour as the flow parameters are changed. Many other aspects of granular impacts are not understood in detail, however, including the complex flows involved in excavating the crater (de Vet et al. 2010) and the physics that leads to the observed scaling of crater dimensions (de Vet \& de Bruyn 2007). In addition, the degree to which the present results apply to other granular systems is unclear, as different granular flows can behave quite differently. Substantial further work will be required to address these challenging issues.

\section{References}

Ambroso, M. A., Kamien, R. D. \& Durian, D. J. 2005 Dynamics of shallow impact cratering. Phys. Rev. E 72, 041305.

Boudet, J. F., Amarouchene, Y. \& Kellay, H. 2006 Dynamics of impact cratering in shallow sand layers. Phys. Rev. Lett. 96, 158001.

Deboeuf, S., Gondret, P. \& Rabaud, M. 2009 Dynamics of grain ejection by sphere impact on a granular bed. Phys. Rev. E 79, 041306.

DE BRUYN, J. R. \& WAlsh, A. M. 2004 Penetration of spheres into loose granular media. Can. J. Phys. 82, 439-446.

DE Vet, S. J. \& DE BRUYn, J. R. 2007 Shape of impact craters in granular media. Phys. Rev. E 76, 041306.

De Vet, S. J., Yohannes, B., Hill, K. M. \& De BruYn, J. R. 2010 Collapse of a rectangular well in a quasi-two-dimensional granular bed. Phys. Rev. E 82, 041304.

Goldman, D. I. \& Umbanhowar, P. 2008 Scaling and dynamics of sphere and disk impact into granular media. Phys. Rev. E 77, 021308.

Katsuragi, H. \& Durian, D. J. 2007 Unified force law for granular impact cratering. Nature Phys. 3, 420-423.

Marston, J. O., Li, E. Q. \& Thoroddsen, S. T. 2012 Evolution of fluid-like granular ejectas generated by sphere impact. J. Fluid Mech. 704, 5-36.

Thoroddsen, S. T., Etoh, T. G., TAKehara, K. \& TAKano, Y. 2004 Impact jetting by a solid sphere. J. Fluid Mech. 499, 139-148.

Thoroddsen, S. T. \& Shen, A. Q. 2001 Granular jets. Phys. Fluids 13, 4-6.

Uehara, J. S., Ambroso, M. A., Ojha, R. P. \& Durian, D. J. 2003 Low-speed impact crater in loose granular media. Phys. Rev. Lett. 90, 194301.

Walsh, A. M., Holloway, K. E., Habdas, P. \& De BRUYn, J. R. 2003 Morphology and scaling of impact craters in granular media. Phys. Rev. Lett. 91, 104301. 